\title{
Análisis Geométrico de un Sistema de Posicionamiento basado en Focos LED y un Array de Fotodiodos
}

\author{
Elena Aparicio-Esteve, Álvaro Hernández, Jesús Ureña \\ Departamento de Electrónica, Universidad de Alcalá \\ elena.aparicio@uah.es
}

\begin{abstract}
Resumen
Los sistemas de posicionamiento local (LPS) basados en luz visible (VLP) son actualmente una alternativa viable, que ha comenzado a expandirse debido a su bajo coste, fácil integración en el entorno de trabajo y ausencia de riesgo para la salud. En la mayoría de los casos, la posición del receptor se obtiene mediante el uso de fotodetectores y técnicas de posicionamiento como triangulación o multilateración, con medidas de tiempos de vuelo, de la atenuación de la señal o del ángulo de llegada. En este trabajo se presenta un análisis geométrico de un sistema de posicionamiento mediante luz visible donde se estudia matemáticamente el comportamiento del array de fotodiodos empleado, así como la influencia de la posición del receptor y de la altura de la apertura en el fotorreceptor QADA en la cobertura del sistema. El sistema propuesto utiliza medidas del ángulo de llegada para estimación de la posición media del receptor. Además, las simulaciones realizadas muestran como óptima la utilización de una apertura a una altura de $2.6 \mathrm{~mm}$
\end{abstract}

Palabras clave: Visible Light Positioning (VLP), luz visible, posicionamiento, array de fotodiodos, focos LED

\section{INTRODUCCIÓN}

En los últimos años, la demanda de un sistema de posicionamiento económico y preciso se ha visto incrementada en gran medida por el desarrollo de distintas aplicaciones y servicios basados en localización, así como por el avance de múltiples tecnologías. Si bien el Sistema de Posicionamiento Global (GPS, Global Positioning System) es el más extendido en la actualidad, su uso se limita a la localización en exteriores principalmente, obteniendo muy buenos resultados en zonas no pobladas. Otros sistemas de posicionamiento como WiFi [3] [13], Bluetooth [12] o radar [14] tratan de suplir las carencias del GPS en espacios donde su precisión disminuye. En relación a los sistemas locales de posicionamiento (LPS, Local Positioning System), se pueden diferenciar los basados en sistemas ópticos, mecánicos, acústicos y de radiofrecuencia, entre otros [25]. Los sistemas de posicionamiento mecánicos son aquellos principalmente basados en Unidades de Medida Inercial (IMU, Inertial Measurement Unit) y suelen combinarse con otros sistemas de posicionamiento [21]. Por otra parte, los sistemas de posicionamiento acústico usan sonidos o ultrasonidos para calcular la posición alcanzando precisiones en el rango de centímetros [16]. Los sistemas de radiofrecuencia permiten posicionar con cualquier señal radio (WiFi; RFID, Radio Frequency Identification; UWB, Ultrawide Band; LTE, LongTerm Evolution...) [17] [25] obteniendo precisiones centimétricas en ciertos casos y aplicaciones muy concretas [2]. Su uso en interiores está muy extendido puesto que ya se dispone de la infraestructura necesaria para su implementación. En último lugar, los sistemas de posicionamiento basados en sistemas ópticos son aquellos que trabajan dentro del espectro de luz visible o infrarroja. Actualmente existe un gran interés en esta tecnología debido a su bajo coste, larga duración, fácil manejo e integración en el entorno de trabajo [26], además de que no supone un riesgo para la salud [8]. Existen numerosos trabajos que utilizan técnicas híbridas combinando los sistemas de posicionamiento mediante luz visible (VLP, Visible Light Positioning) con ultrasonidos [19] o WiFi [11], entre otros, para aportar mayor robustez y precisión al enlace.

Los sistemas VLP se utilizan tanto para posicionar en exteriores como en interiores. En términos generales, la mayoría de trabajos se centran en posicionamiento en interiores debido a la gran influencia que tiene la luz ambiental en este tipo de sistemas [8]. En el caso de entornos interiores, si bien el transmisor es normalmente una lámpara o array de LEDs (Light Emitting Diode), en recepción se pueden distinguir sistemas de posicionamiento basados en sensores de imagen y aquellos basados en fotodetectores. Los sistemas de posicionamiento basados en sensores de imagen utilizan la cámara de un teléfono móvil convencional [20] o cámaras CMOS [7] [5]. No obstante, requieren de algoritmos de procesado de imagen que ralentizan el cálculo de la posición [18]. Los sistemas de posicionamiento cuyo receptor es un fotodetector hacen uso de fotodiodos [1] [27] o un array de fotodiodos [4] [29], los cuales, ante el 
estímulo de la luz, generan una corriente a partir de la cual es posible estimar la posición del receptor mediante la aplicación de algún algoritmo de posicionamiento. Entre ellos destaca el uso de Quadrant Photodiode Angular Diversity Aperture (QADA) [4]. La técnica de posicionamiento más utilizada en estos casos es la triangulación o multilateración con medidas de tiempos de vuelo (TOA, Times-of-Arrival; TDOA, Time-Difference-ofSrrival) [27], medidas de la atenuación de la señal (RSS, Received Signal Strength) [10] [22] o medidas por ángulo de llegada (AOA, Angle-of-Arrival) [26]; aunque también se utilizan otros métodos como por proximidad [28]. Las principales desventajas del cálculo de medidas de tiempos de vuelo son la sincronización requerida y la velocidad de la luz, la cual provoca que errores en la estimación del tiempo de $1 \mathrm{~ns}$ conlleven un error de $30 \mathrm{~cm}$ en la posición [6]. El posicionamiento mediante RSS supone tener en cuenta las reflexiones de la luz en las distintas superficies y el consiguiente efecto multicamino, incrementando la dificultad del mismo [6]. Finalmente, el algoritmo de posicionamiento basado en AOA parte de la estimación de los ángulos de llegada procedentes de varios LEDs. Su principal ventaja es que tan solo requiere de tres medidas para posicionar en tres dimensiones, además de que no necesita sincronización entre los transmisores [6].

Este trabajo se centra en la definición y estudio de un sistema de posicionamiento en interiores. Éste se basa en el uso de tres lámparas LED como transmisores y un array de fotodiodos como receptor. La técnica empleada para el posicionamiento del receptor es la triangulación y el cálculo de la distancia entre transmisor y receptor se realiza mediante los ángulos de llegada (AOA). Más concretamente, este estudio se centra en analizar geométricamente dicho sistema de posicionamiento, evaluando los distintos parámetros de configuración del mismo y su influencia sobre las prestaciones finales alcanzadas. El resto del documento se encuentra organizado del siguiente modo: la sección 2 realiza una descripción de los emisores LED y del array de fotodiodos propuestos; en la sección 3 se detalla la geometría del sistema de posicionamiento empleada; la sección 4 muestra las simulaciones realizadas, evaluando la influencia de la posición del receptor en relación a los focos, así como otros parámetros geométricos de la propuesta; y, finalmente, en la sección 5 se abordan las conclusiones.

\section{DESCRIPCIÓN GENERAL DEL SISTEMA PROPUESTO}

El sistema de posicionamiento propuesto se basa en un conjunto de emisores/focos LED (al menos tres) que se ubicarán en determinadas posiciones del espacio, de forma que iluminen un área común de cobertura, dentro de la cual un receptor pueda detectar las emisiones y estimar su posición como se aprecia en la figura 1 .

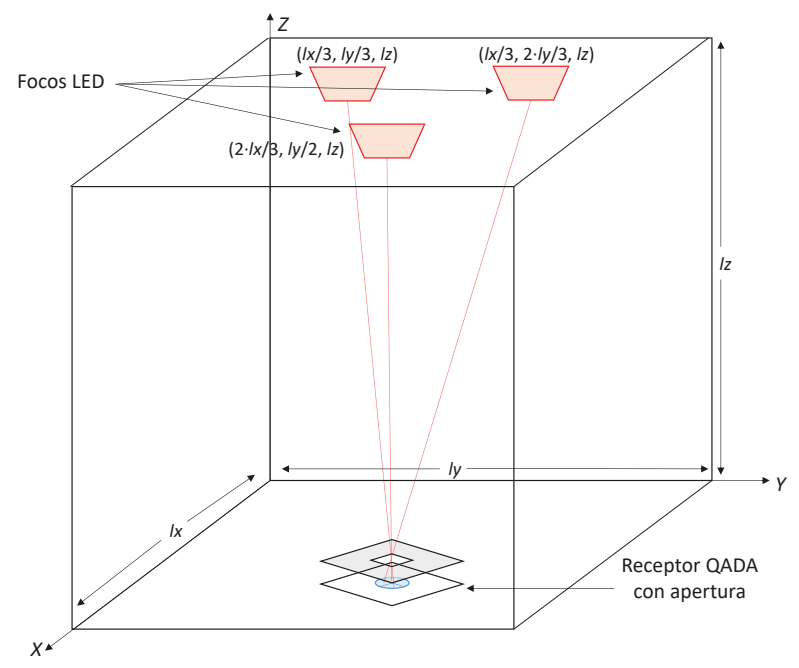

Figura 1: Sistema de posicionamiento con emisores LED y array de fotodiodos.

Los emisores LED empleados en este trabajo constan cada uno de un total de ocho módulos de la serie OVM12F3x7 [24], conteniendo cada módulo tres LEDs. En concreto, los módulos considerados son el OVM12F3W7 (color blanco, luminosidad típica de 16lux@0.5m, 5000-7000K) y el OVM12F3R7 (color rojo, luminosidad típica de 20lux@0.5m, $\lambda=623+/-$ $3 \mathrm{~nm})$. Ambos tienen un ángulo de haz de $120^{\circ}$, emitiendo un máximo de $1.5 \mathrm{~W}$ ante un voltaje de entrada típico de $12 \mathrm{~V}$. Estos módulos LED están conectados con la tarjeta TPS92515HVEVM-749 [23], que incluye el driver tipo buck TPS92515QDGQRQ1 para LEDs, el cual además permitirá en trabajos futuros la aplicación de técnicas de modulación y codificación en los distintos focos.

Por otra parte, el receptor empleado es el QP50-6-18uSD2 [9]. Consta de un fotorreceptor circular QADA QP50-6-18u-TO8 de radio $r=3.9 \mathrm{~mm}$, formado por un array de cuatro fotodiodos, cada uno de ellos con un área efectiva de $11.78 \mathrm{~mm}^{2}$. El módulo tiene como salidas la diferencia de voltajes entre los cuatro fotodiodos, tanto en eje $X$ como en eje $Y$, así como la suma total del voltaje incidente. Su responsividad a una longitud de onda de $\lambda=625 \mathrm{~nm}$ es de $0.4 \mathrm{~A} / \mathrm{W}$ y el ruido teórico es de $15 \mathrm{nV} / \sqrt{ } \mathrm{Hz}$ ante una entrada de $15 \mathrm{~V}$. $\mathrm{Al}$ array de fotodiodos se le ha añadido una apertura cuadrada realizada mediante impresión 3-D, de lado igual al diámetro del círculo del fotorreceptor $(7.8 \mathrm{~mm})$ y situada a una altura $h_{a p}=2.6 \mathrm{~mm}$ sobre el mismo. 


\section{GEOMETRÍA DEL SISTEMA DE POSICIONAMIENTO PROPUESTO}

El sistema de posicionamiento propuesto en este trabajo consta por un lado del receptor QADA descrito anteriormente, colocado directamente bajo una apertura cuadrada según se muestra en la figura 3.a. En la figura 2 se observan los cuatro cuadrantes y la zona cubierta por el haz de luz que atraviesa la apertura para el caso de un rayo incidente en el punto $(x, y)$.

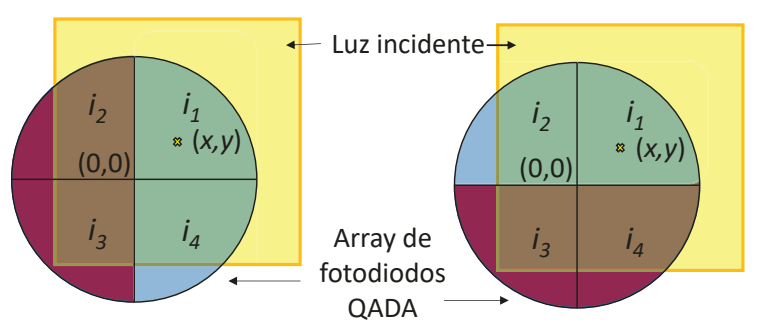

Figura 2: Incidencia de la luz en el receptor QADA a través de la apertura para ambas coordenadas: eje $X$ (izquierda), eje $Y$ (derecha).

Por otro lado, aunque el sistema de posicionamiento requiere el uso de al menos tres focos LED para poder estimar la posición del receptor (véase la figura 1), en aras de una mayor simplicidad en la explicación, se va a proceder al análisis geométrico del sistema de posicionamiento para el caso de transmitir con un único foco/transmisor LED. Posteriormente, el análisis podrá ser extendido con facilidad al resto de transmisores LED.

El principio de funcionamiento de la propuesta se basa en el hecho de que la luz proveniente de los emisores atraviesa el centro de la apertura cuadrada colocada sobre el receptor, e incide en el array de fotodiodos (véase la figura 3.a). Las coordenadas $(x, y)$ del punto de impacto de la luz incidente dentro de la superficie del receptor QADA permiten estimar el ángulo de incidencia $\psi$ del haz. Según este ángulo $\psi$, se iluminan distintas áreas en los cuatro fotodiodos que forman el receptor. De esta forma, se pueden definir los ratios $p_{x}(t)$ y $p_{y}(t)$, en función de las corrientes $i_{j}(t)$ para cada cuadrante $j=1,2,3,4[4]$.

$$
\begin{aligned}
& p_{x}(t)=\frac{i_{1}(t)+i_{4}(t)}{i_{2}(t)+i_{3}(t)} \\
& p_{y}(t)=\frac{i_{1}(t)+i_{2}(t)}{i_{3}(t)+i_{4}(t)}
\end{aligned}
$$

Teniendo en cuenta que dichos ratios en ausencia de ruido son proporcionales a las áreas $A_{j}(t)$ de los distintos cuadrantes, las ecuaciones (1) y (2) se pueden expresar como (3) y (4):

$$
\begin{aligned}
& p_{x}(t)=\frac{A_{1}(t)+A_{4}(t)}{A_{2}(t)+A_{3}(t)} \\
& p_{y}(t)=\frac{A_{1}(t)+A_{2}(t)}{A_{3}(t)+A_{4}(t)}
\end{aligned}
$$
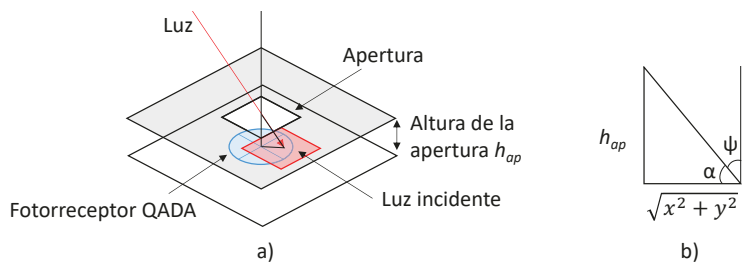

b)

Figura 3: a) Incidencia de la luz en el fotorreceptor QADA; b) análisis geométrico de la apertura.

A continuación se detalla el cálculo de las distintas áreas $A_{j}(t)$ para $p_{x}(t)$ en el primer cuadrante, $x, y \in[0, r]$ (5-9), donde $r=3.9 \mathrm{~mm}$ es el radio del fotorreceptor. Es posible realizar el análisis análogo para $p_{y}(t)$. Nótese que el tercer cuadrante tiene un área $A_{3}(t)$ que depende de la posición en la que se encuentre la apertura con respecto al fotorreceptor, tal y como se observa en la figura 4 y se detalla en (7) y (8) para los dos casos $A_{3, I}(t)$ y $A_{3,2}(t)$, respectivamente.

$$
\begin{gathered}
A_{1}(t)=\frac{\pi \cdot r^{2}}{4} \\
A_{2}(t)=\frac{\pi \cdot r^{2}}{4}-\frac{1}{2} \cdot\left[\frac{r^{2}}{2} \cdot(\theta-\sin (\theta))\right] \\
A_{3,1}(t)=(r-x) \cdot(r-y) \\
A_{3,2}(t)=\frac{\pi \cdot r^{2}}{4}-\frac{1}{2} \cdot\left[\frac{r^{2}}{2} \cdot(\theta-\sin (\theta))\right] \\
-\frac{1}{2} \cdot\left[\frac{r^{2}}{2} \cdot\left(\theta^{\prime}-\sin \left(\theta^{\prime}\right)\right)\right] \\
A_{4}(t)=\frac{\pi \cdot r^{2}}{4}-\frac{1}{2} \cdot\left[\frac{r^{2}}{2} \cdot\left(\theta^{\prime}-\sin \left(\theta^{\prime}\right)\right)\right]
\end{gathered}
$$

Siendo $r$ el radio del receptor; y $\theta=2 \cdot \operatorname{acos}\left(1-\frac{x}{r}\right)$ y $\theta^{\prime}=2 \cdot \operatorname{acos}\left(1-\frac{y}{r}\right)$ los ángulos del segmento circular. Se aprecia que el área $A_{l}(t)$ no depende ni de $x$ ni de $y, A_{2}(t)$ solo depende de $x, A_{4}(t)$ solo depende de $y$, mientras que $A_{3}(t)$ depende tanto de $x$ como de $y$. 


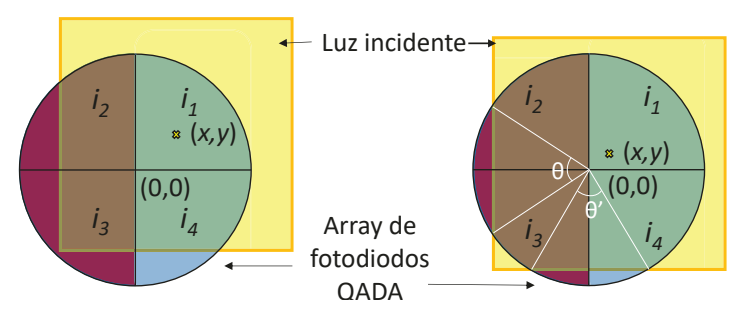

Figura 4: Incidencia de la luz en el receptor QADA a través de la apertura en los casos del área $A_{3, I}(t)$

(izquierda) y $A_{3,2}(t)$ (derecha).

En la figura 5 se muestra la representación del ratio $p_{x}(t)$ (3) en función de $x$ para distintos valores de $y$. Nótese que las curvas obtenidas para los distintos valores de y están prácticamente superpuestas, por lo que la dependencia en $y \in[0, r]$ es mínima.

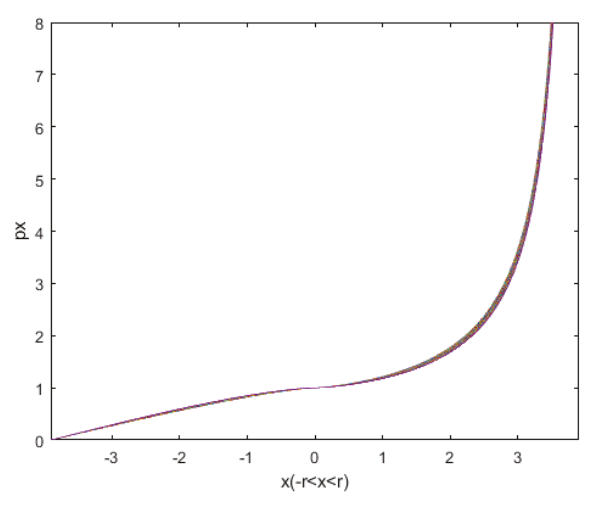

Figura 5: Ratio $p_{x}(t)$ en función de la coordenada $x$ para distintos valores de $y ; x \in[-r, r], y \in[0, r]$.

Las salidas proporcionadas por el receptor QP50-6$18 \mathrm{u}-\mathrm{SD} 2$ son la combinación de las corrientes $i_{j}(t)$ obtenidas en los distintos fotodiodos, transformadas en voltajes $v_{j}(t)$ mediante un amplificador de transimpedancia, de forma que las ecuaciones (3) y (4) resultan en (10) y (11):

$$
\begin{aligned}
& p_{x}(t)=\frac{A_{1}(t)+A_{4}(t)}{A_{2}(t)+A_{3}(t)}=\frac{V_{\text {sum }}-V_{L R}}{V_{\text {sum }}+V_{L R}}, \forall x \\
& p_{y}(t)=\frac{A_{1}(t)+A_{2}(t)}{A_{3}(t)+A_{4}(t)}=\frac{V_{\text {sum }}-V_{B T}}{V_{\text {sum }}+V_{B T}}, \forall y
\end{aligned}
$$

Siendo $V_{\text {sum }}=\sum_{j=1}^{4} v_{j}(t) ; V_{L R}=\left(v_{2}(t)+v_{3}(t)\right)-$ $\left(v_{1}(t)+v_{4}(t)\right) ; \quad$ у $\quad V_{B T}=\left(v_{3}(t)+v_{4}(t)\right)-$ $\left(v_{1}(t)+v_{2}(t)\right)[9]$.

En la propuesta realizada en este trabajo las coordenadas $x(t)$ e $y(t)$ se calculan mediante la gráfica mostrada en la figura 5 para el ratio $p_{x}(t)$, y de manera similar para $p_{y}(t)$.

Una vez calculada la posición $(x, y)$ de incidencia del haz de luz, se procede a la estimación de la posición del receptor QADA dentro del área de cobertura. Dicho cálculo se realiza geométricamente mediante la relación existente entre la altura $h_{a p}$, a la que se encuentra la apertura con respecto a los fotodiodos del receptor, y la posición $(x, y)$ de incidencia del haz de luz, siguiendo el esquema mostrado en la figura 3 . Para una mayor resolución se requiere el uso completo del área de los fotodiodos, por lo que se define el lado del cuadrado que compone la apertura igual al diámetro del fotorreceptor (véanse las figuras 2 y 3.a).

El cálculo del ángulo de incidencia $\psi$ se puede realizar según (12) y (13).

$$
\begin{gathered}
\alpha=\operatorname{tag}^{-1}\left(\frac{h_{a p}}{\sqrt{x^{2}+y^{2}}}\right) \\
\psi=90^{\circ}-\alpha
\end{gathered}
$$

Donde $\alpha$ es el ángulo con el que el haz llega al receptor; $\psi$ es el ángulo con el que el haz de luz que pasa por el centro de la apertura incide en el array de fotodiodos (véase la figura 3.b); $x$ e $y$ son las posiciones en las que se encuentra focalizado el haz de luz procedente del foco LED (punto de incidencia); y $h_{a p}$ es la altura a la que se encuentra la apertura cuadrada, mencionada anteriormente. A partir del ángulo de incidencia $\psi$ se puede establecer la distancia $d$ entre transmisor y receptor (14).

$$
d=\frac{H}{\cos (\psi)}
$$

En la figura 6 se presentan los parámetros utilizados en (14), siendo $d$ la distancia entre transmisor y receptor y $H$ la altura a la que se encuentra el emisor LED con respecto al receptor.

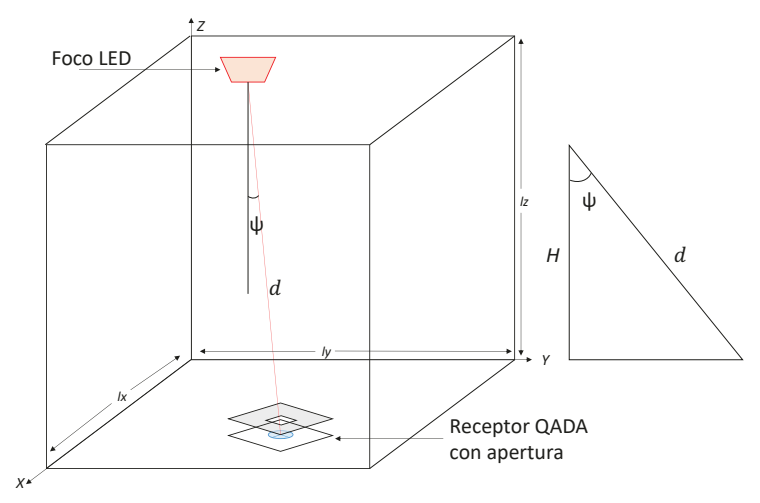

Figura 6: Escenario del análisis geométrico de la distancia $H$ entre transmisor y receptor.

Una vez conocida la distancia absoluta entre transmisor y receptor es posible estimar la posición del receptor mediante el algoritmo de posicionamiento Gauss-Newton esférico [15]. 


\section{4}

\section{ANÁLISIS DE LA INFLUENCIA DE LA POSICIÓN DEL RECEPTOR CON RESPECTO A LOS FOCOS}

Se pasa a continuación a analizar los distintos parámetros geométricos de la propuesta realizada, y la influencia que éstos pueden tener en el funcionamiento final del sistema. En primer lugar, la posición de los focos LED se establece de forma que cubran la mayor superficie posible para un determinado espacio. En la tabla 1 se aprecian las coordenadas $(x, y, z)$ definidas para los focos LED, atendiendo a este criterio, donde $l_{x}, l_{y}$ y $l_{z}$ son las dimensiones de un espacio elegido como ejemplo para las siguientes simulaciones, con unas dimensiones de $2 \times 2 \times 2 m$.

Tabla 1: Coordenadas de los focos LED empleadas en este estudio.

\begin{tabular}{|c|c|}
\cline { 2 - 2 } \multicolumn{1}{c|}{} & Coordenadas $(\boldsymbol{x}, \boldsymbol{y}, \boldsymbol{z})$ \\
\hline LED 1 & $\left(l_{x} / 3, l_{y} / 3, l_{z}\right)$ \\
LED 2 & $\left(l_{x} / 3,2 \cdot l_{y} / 3, l_{z}\right)$ \\
LED 3 & $\left(2 \cdot l_{x} / 3, l_{y} / 2, l_{z}\right)$ \\
\hline
\end{tabular}

Se realiza a continuación un análisis para determinar la altura $h_{a p}$ óptima de la apertura para un único transmisor, LED 1, siendo éste y el LED 2 los casos más restrictivos, puesto que deben cubrir una mayor superficie. Se evalúan las posibles alturas $h_{a p}$ de la apertura desde 0 hasta $8 \mathrm{~mm}$ para todo el espacio $2 \times 2 \times 2 \mathrm{~m}$, siendo la única restricción la obtención de un radio del fotodetector menor o igual a $3.9 \mathrm{~mm}$ para asegurar la capacidad de posicionamiento en dicha posición (recuérdese que el radio del fotodetector QADA es de $3.9 \mathrm{~mm}$ ). El análisis se ha realizado para las posiciones del receptor en el recorrido diagonal desde la proyección horizontal del transmisor hasta el punto más alejado de la habitación sobre un mismo plano horizontal $z=$ cte., como se muestra en la figura 7 en verde, donde hay una distancia $d$ máxima de $1.2019 \mathrm{~m}$.

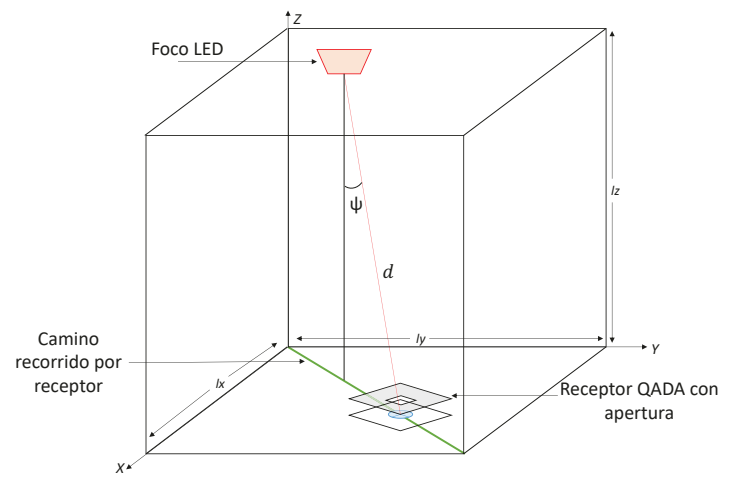

Figura 7: Escenario de simulación para la obtención de la altura $h_{a p}$ de apertura óptima.
La figura 8 representa el rango de valores en los que incide el haz de luz para distintas alturas $h_{a p}$ de la apertura. Este análisis se ha realizado para planos horizontales en los cuales se ubica el receptor $(z=0$, $0.5 \mathrm{~m}, 1 \mathrm{~m}, 1.5 \mathrm{~m})$, siendo $z=0 \mathrm{~m}$ la curva exterior $\mathrm{y}$ $z=1.5 \mathrm{~m}$ la curva interior.

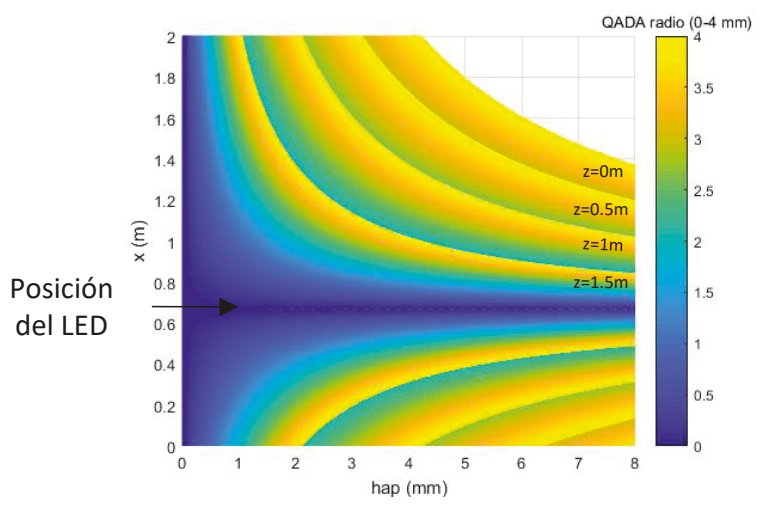

Figura 8: Comparación de la incidencia de la luz a distintas alturas de la apertura $h_{a p}$, en función de distintos planos horizontales $(z=0,0.5 \mathrm{~m}, 1 \mathrm{~m}, 1.5 \mathrm{~m})$ de ubicación del receptor.

En aras de una mayor claridad, se muestran a continuación en las figuras 9 y 10 de forma individual las gráficas correspondientes al análisis para el plano $z=0 \quad \mathrm{y} \quad z=1 \mathrm{~m}$. Nótese que estos dos casos se corresponden con una distancia $d$ entre el transmisor LED y el receptor de $2 \mathrm{~m}$ y $1 \mathrm{~m}$, respectivamente.

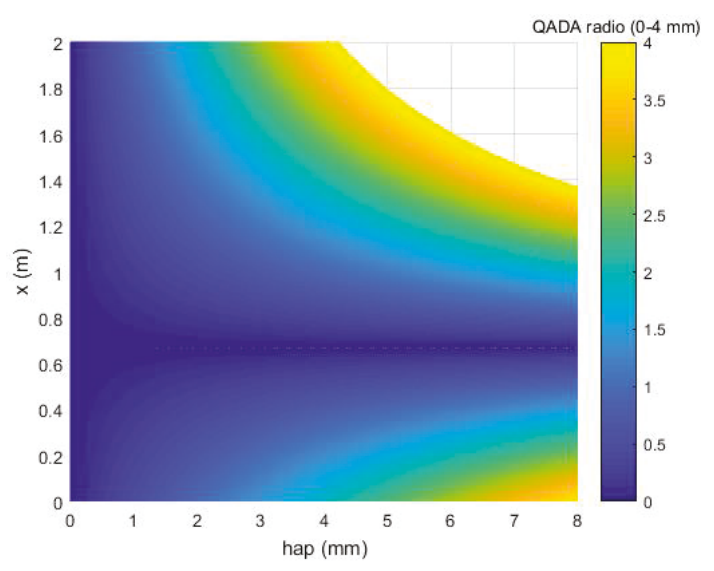

Figura 9: Análisis particular de la incidencia de la luz a distintas alturas $h_{a p}$ de la apertura para el plano $\mathrm{z}=0$ del receptor. 


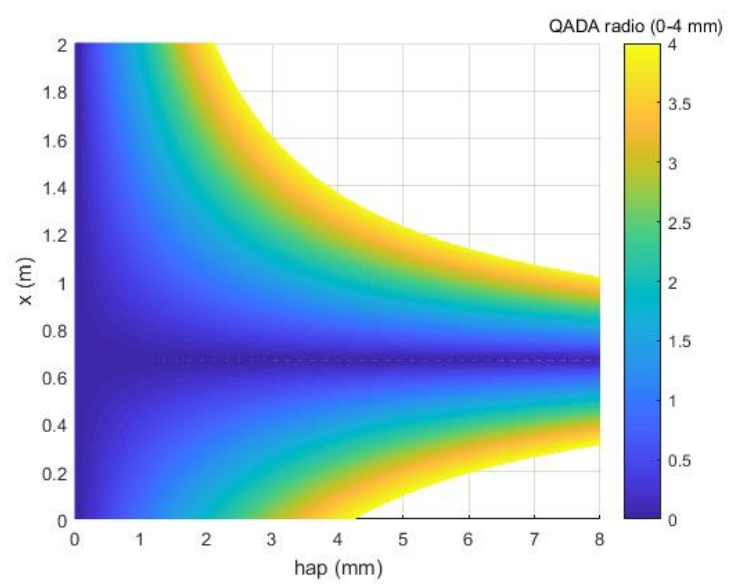

Figura 10: Análisis particular de la incidencia de la luz a distintas alturas $h_{a p}$ de la apertura para el plano $z=1 \mathrm{~m}$ del receptor.

En las figuras 8, 9 y 10 se aprecia un gran rango donde los puntos de incidencia del haz de luz recibidos en el fotodetector QADA están muy próximos entre sí, coincidiendo con la localización del transmisor en $x=0.67 \mathrm{~m}$. Este hecho se agrava a medida que disminuye la altura $z$ a la cual se encuentra el receptor (aumenta la distancia $d$ entre transmisor y receptor), y/o disminuye la altura $h_{a p}$ a la que se encuentra la apertura. Por otra parte, una baja altura $h_{a p}$ de la apertura supone una gran zona de cobertura. Con el fin de disponer de la mayor zona de cobertura posible, se decide escoger una altura de la apertura de $h_{a p}=2.6 \mathrm{~mm}$, la distancia mínima a la que se puede colocar la apertura cuadrada en el fotodetector por restricciones de su diseño mecánico.

Con esta configuración, se ha procedido a evaluar la zona de cobertura para las posiciones de los focos LED indicadas en la tabla 1. En la figura 11 se muestra las zonas de cobertura para cada uno de los focos con los parámetros indicados en la tabla 2 .

Tabla 2: Parámetros de diseño empleados en este estudio.

\begin{tabular}{|c|c|}
\hline Parámetro & Valor \\
\hline Altura $h_{a p}$ & $2.6 \mathrm{~mm}$ \\
Radio QADA & $3.9 \mathrm{~mm}$ \\
Volumen & $2 \times 2 \times 2 \mathrm{~m}$ \\
FOV tx & $120^{\circ}$ \\
\hline
\end{tabular}

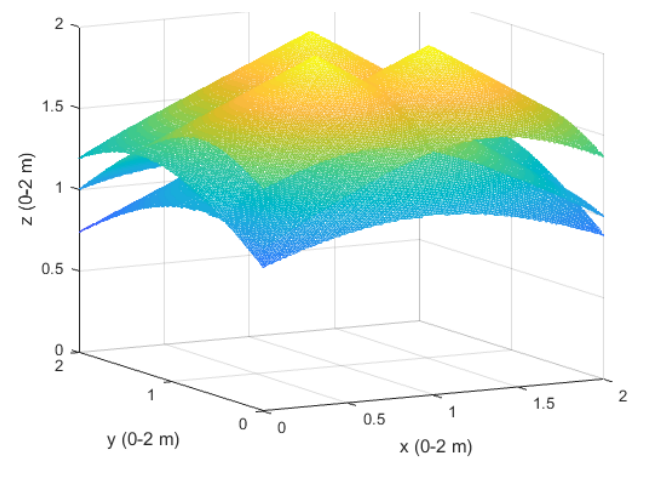

Figura 11: Zona de cobertura para cada uno de los focos LED ubicados en el espacio definido para este estudio.

Por otro lado, la figura 12 muestra la zona de cobertura donde es posible posicionar el receptor, es decir, la zona de cobertura conjunta de los tres focos LED considerados. Se aprecia que, a excepción de las esquinas del espacio, donde el rango de cobertura disminuye ligeramente, se consigue cubrir todos los puntos cuya altura $z$ sea inferior a $1 \mathrm{~m}$, llegando a alcanzar una cobertura máxima en el centro de la habitación de 1.75 metros.

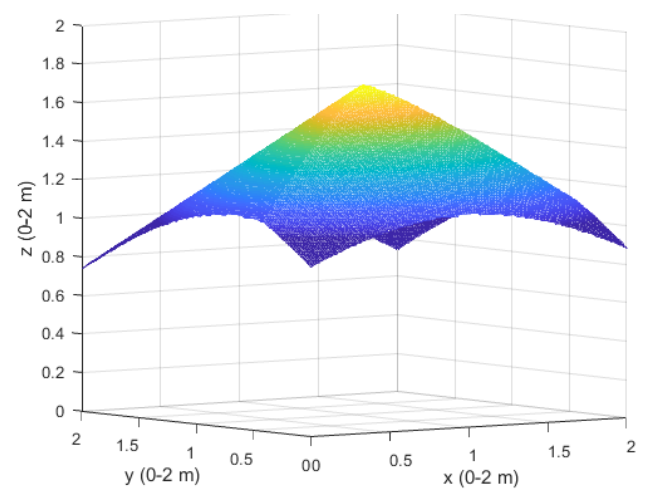

Figura 12: Zona de cobertura de posicionamiento conjunta para los tres focos LED considerados en este estudio.

\section{CONCLUSIONES}

En este trabajo se ha realizado un análisis geométrico de un sistema de posicionamiento basado en luz visible, el cual consta de un array de fotodiodos como receptor, y un total de tres focos LED. La propuesta ha sido analizada mediante simulación para un espacio con unas dimensiones de $2 \times 2 \times 2 \mathrm{~m}$, de cara a definir de forma adecuada la altura de la apertura implementada sobre el fotorreceptor QADA. Igualmente se ha analizado el mapa de cobertura resultante, determinando los límites del mismo. En dichas condiciones se obtiene, con una altura de la apertura de $2.6 \mathrm{~mm}$, una zona de cobertura completa para todo el espacio considerado cuando el receptor se encuentra 
en una altura inferior a $0.8 \mathrm{~m}$. Conviene destacar que la cobertura en la zona central de la habitación alcanza hasta una altura de $1.75 \mathrm{~m}$ en su punto central.

En futuros trabajos se abordará la posible influencia de la luz ambiental en los cálculos realizados, especialmente en los extremos de la zona de estudio, las posibles interferencias entre los distintos focos existentes, así como las restricciones que puedan surgir de un funcionamiento propio de un entorno real.

\section{Agradecimientos}

Este trabajo ha sido posible gracias al Ministerio de Economía, Industria y Competitividad (proyecto SOC-PLC, ref. TEC2015-64835-C3-2-R, y proyecto TARSIUS, ref. TIN2015-71564-C4-1-R).

\section{English summary}

\section{GEOMETRICAL ANALYSIS OF A POSITIONING SYSTEM BASED ON LED LAMPS AND A PHOTODIODE ARRAY}

\begin{abstract}
Local Positioning Systems (LPS) based on visible light (VLP) are, nowadays, a reliable alternative which is being developed due to its low cost, environmentfriendly and the absence of any risk to human health. The receiver positioning is obtained mainly by using photodetectors and positioning techniques such as triangulation or multilateration, through times-offlight measurements, signal attenuation measurements or angle-of-arrival measurements. This work geometrically analyzes a positioning system based on visible light. The behavior of the photodiode array used has been mathematically studied, as well as the influence from the receiver position and from the QADA aperture height on the system coverage map. The proposed positioning system uses angles-ofarrival measurements in order to estimate an averaged receiver position. Simulations provides as optimal the use of a $2.6 \mathrm{~mm}$ aperture height.
\end{abstract}

Keywords: Visible Light Positioning (VLP), visible light, positioning, photodiode array, LED lamps.

\section{Referencias}

[1] Cai, Y., Guan, W., Wu, Y., Xie, C., Chen, Y., and Fang, L., (2017) "Indoor High Precision ThreeDimensional Positioning System Based on Visible Light Communication Using Particle
Swarm Optimization", IEEE Photonics Journal, vol. 9(6), pp. 1-20.

[2] Chen, C., Chen, Y., Han, Y., Lai, H. Q., Zhang, F., and Liu, K. J. R., (2017) "Achieving CentimeterAccuracy Indoor Localization on WiFi Platforms: A Multi-Antenna Approach", IEEE Internet of Things Journal, vol. 4(1), pp. 122134.

[3] Chen, C., Han, Y., Chen, Y., and Liu, K. J. R., (2016) "Indoor GPS with centimeter accuracy using WiFi", 2016 Asia-Pacific Signal and Information Processing Association Annual Summit and Conference (APSIPA), pp. 1-4.

[4] Cincotta, S., He, C., Neild, A., and Armstrong, J., (2018) "High angular resolution visible light positioning using a quadrant photodiode angular diversity aperture receiver (QADA)", Optics Express, vol. 26(7), pp. 9230-9242.

[5] Danakis, C., Afgani, M., Povey, G., Underwood, I., and Haas, H., (2012) "Using a CMOS camera sensor for visible light communication", Proceedings of the Globecom Workshops (GC Wkshps), pp. 1244-1248.

[6] Do, T-H., and Yoo, M., (2016) "An in-Depth Survey of Visible Light Communication Based Positioning Systems", Sensors 16(5), no. 678, pp $1-40$.

[7] Do, T. H., and Yoo, M., (2016) "Performance Analysis of Visible Light Communication Using CMOS Sensors", Sensors, vol. 16(3), no. 309, pp. 1-23.

[8] Do, T. H., and Yoo, M., (2015) "Potentialities and challenges of VLC based outdoor positioning", 2015 International Conference on Information Networking (ICOIN), pp. 474-477.

[9] First Sensor Inc., (2012) Series 6 Data Sheet Quad Sum and Difference Amplifier, Part Description QP50-6-18u-SD2, Product Specification.

[10] Gonendik, E., and Gezici, S., (2015) "Fundamental Limits on RSS Based Range Estimation in Visible Light Positioning Systems", IEEE Communications Letters, vol. 19(12), pp. 2138-2141.

[11] Huang, Q., Zhang, Y., Ge, Z., and Lu, C., (2015) "Refining Wi-Fi Based Indoor Localization with Li-Fi Assisted Model Calibration in Smart Buildings", Proceedings of the 2016 International Conference on Computing in Civil and Building Engineering, pp. 1-8. 
[12] Kalbandhe, A. A., and Patil, S. C., (2016) "Indoor Positioning System using Bluetooth Low Energy", 2016 International Conference on Computing, Analytics and Security Trends (CAST), pp. 451-455.

[13] Kovalev, M., (2014) "Indoor positioning of mobile devices by combined Wi-Fi and GPS signals", 2014 International Conference on Indoor Positioning and Indoor Navigation (IPIN), pp. 332-339.

[14] Lee, R. H., Wu, J. C., Chang, S. H., Chang, S. F., Chang, C. C., and Chen, Y. M., (2013) "Radar design for wireless indoor positioning applications", 2013 European Microwave Conference, pp. 846-849.

[15] Li, X., and Scaglione, A., (2013) "Convergence and applications of a gossip-based gauss-newton algorithm," IEEE Trans. Signal Process., vol. 61(21), pp. 5231-5246

[16] Lindo, A., Garcia, E., Ureña, J., del Carmen Perez, M., Hernandez, A., (2015) "Multiband waveform design for an ultrasonic indoor positioning system", IEEE Sensors Journal, vol. 15(12), pp. 7190-7199.

[17] Mikšaj, U. T., and Bonefačić, D., (2016) "Propagation characteristics of UHF radiofrequency identification system signal for application in indoor positioning", 2016 22nd International Conference on Applied Electromagnetics and Communications (ICECOM), pp. 1-6.

[18] Pathak, P. H., Feng, X., Hu, P., and Mohapatra, P., (2015) "Visible Light Communication, Networking, and Sensing: A Survey, Potential and Challenges," IEEE Communications Surveys \& Tutorials, vol. 17(4), pp. 2047-2077.

[19] Rabadan, J., Guerra, V., Rodríguez, R., Rufo, J., Luna-Rivera, M., and Perez-Jimenez, R., (2017) "Hybrid Visible Light and Ultrasound-Based Sensor for Distance Estimation". Sensors, vol. 17(2), no. 330, pp. 1-9.

[20] Rajagopal, N., Lazik, P., Rowe, A., (2014) "Visual light landmarks for mobile devices", Proceedings of the $13^{\text {th }}$ International Symposium on Information Processing in Sensor Networks, pp. 249-260.

[21] Ruiz, A. R. J., Granja, F. S., Honorato, J. C., and Rosas J. I., (2010) "Pedestrian indoor navigation by aiding a foot-mounted IMU with RFIDsignal strength measurements", Proc. Int. Conf. Indoor Positioning Indoor Navigat.(IPIN), pp. 1-7.

[22] Steendam, H., Wang, T. Q., and Armstrong, J., (2017) "Theoretical Lower Bound for Indoor Visible Light Positioning Using Received Signal Strength Measurements and an Aperture-Based Receiver", Journal of Lightwave Technology, vol. 35(2), pp. 309-319.

[23] Texas Instruments Inc., (2016) TPS92515HVEVM-749 High Current Buck LED Driver Evaluation Module, User's Guide.

[24] TT Electronics/Optek Technology, (2016) 3 LED Module (30 modules/string) OVM12F3×7 series, Product Specification.

[25] Ureña, J., Hernández, A., García, J.J., Villadangos, J. M., Pérez, M. C., Gualda, D., Álvarez, F. J., and Aguilera, T., (2018) "Acoustic Local Positioning With Encoded Emission Beacons", Proceedings of the IEEE, vol. 106(6), pp. 1042-1062.

[26] Wang, C., Wang, L., Chi, X., Liu, S., Shi, W., and Deng, J., (2015) "The research of indoor positioning based on visible light communication", China Communications, vol. 12(8), pp. 85-92.

[27] Wang, T. Q., Sekercioglu, Y. A., Neild, A., and Armstrong, J., (2013) "Position Accuracy of Time-of-Arrival Based Ranging Using Visible Light With Application in Indoor Localization Systems", Journal of Lightwave Technology, vol. 31(20), pp. 3302-3308.

[28] Xie, C., Guan, W., Wu, Y., Fang, L., and Cai, Y., (2018) "The LED-ID Detection and Recognition Method Based on Visible Light Positioning Using Proximity Method", IEEE Photonics Journal, vol. 10(2), pp. 1-16.

[29] Yang, S. H., Kim, H. S., Son, Y. H., and Han, S. K., (2014) "Three-Dimensional Visible Light Indoor Localization Using AOA and RSS With Multiple Optical Receivers", Journal of Lightwave Technology, vol. 32(14), pp. 24802485.

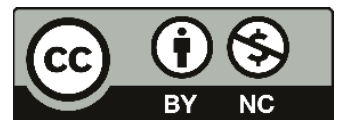
(C) 2018 by the authors. Submitted for possible open access publication under the terms and conditions of the Creative Commons Attribution CC-BY-NC $\quad 3.0 \quad$ license (https://creativecommons.org/licenses/by-nc/3.0). 